\title{
Research of combination of electricity GM $(1,1)$ and seasonal time series forecasting model
}

\author{
Wang Yuping ${ }^{1}$, Zheng Fangpeng ${ }^{2}$, He Kelei ${ }^{3}$, Li Chunxue ${ }^{4}$ \\ ${ }^{1}$ Guizhou Power Grid Corporation,Guiyang,Guizhou,P.R.C \\ ${ }^{2}$ Guizhou Power Grid Corporation,Guiyang,Guizhou,P.R.C \\ ${ }^{3}$ North China Electric Power University,Beijing,P.R.C \\ ${ }^{4}$ North China Electric Power University,Beijing,P.R.C
}

KEY WORD: load forecasting; GM $(1,1)$; seasonal time series; combination forecasting model

ABSTRACT: Load forecasting is one of the important content of power system. Gray GM $(1,1)$ model with seasonal time series was proposed by analyzing the development of the electricity load forecasting. This paper made an empirical analysis of the model with data specifically related to electricity in Guizhou region in recent years. The results showed that the proposed method has a higher accuracy than a single prediction model, and proved correctness and effectiveness of the combination forecasting model.

\section{INTRODUCTION}

With the power entering the market operation, the power load forecasting is forecasting the electricity market demand in essence. As the power supply has the characteristic of instantaneous balance, the power industry forecasting is more urgent than any other industries. Lower power load forecasting can not meet the electricity demand; higher load forecasting will reduce the operating efficiency of some generation and transmission equipment, and it will cause unnecessary wasted investment. Therefore, it has great significance to do well in the power load forecasting on the realization of the grid security and economic operation ${ }^{[1]}$. In literature [2], Grey Prediction Model is used to predict the power load of Wing city, and then the forecasting results are analyzed. Literature [3] proposed an electric power load forecasting model based on GM $(1,1)$ model, and then the feasibility of this model in the electric power load forecasting is proved through examples. In order to improve the prediction accuracy of gray prediction $\operatorname{GM}(1,1)$, automatic optimization setting weight is proposed and used to choose background values. And the initial value of the gray prediction GM $(1,1)$ model is improved based on the principle of least squares method. In literature [5], a load forecasting method based on chaotic time series is used to forecast monthly electricity consumption. Literature [6] proposes a power system load forecasting method based on the combination of time series and neural networks. This method predicts power load through a lot of historical data random sequence,and the forecasting result will be corrected by neural network. Literature [7] proposes a short-term load forecasting method based on a back propagation artificial neural network. Then an auto-correlation analysis method is used, and a short-term load forecasting method is established for Shanxi power based on BP-ANN. In literature [8], a fuzzy evaluation method is used to assign relative weight for each predicting model, and then a new combination forecasting model is established for power load forecasting. Literature [9] proposes a combination forecasting method of timing analysis and neural network. It can take advantage of different models to handle different parts of the data so that more accurate predictions can be obtained. According to the characteristics analysis of the small grid load data, with the combination of time sequence processing, chaos theory and neural network theory, a power load forecasting model is proposed based on chaotic neural network theory. Currently, many researches on load forecasting neglect the local electricity characteristics. And the methods proposed are not very practical. In this context, according to the load characteristics of Guizhou, this paper proposes an electricity load forecasting model with the combination of gray prediction GM $(1,1)$ model and seasonal time series. It make full use of the respective advantages of gray prediction GM $(1,1)$ model and seasonal time series. And then the feasibility and effectiveness of this model is verified through empirical analysis. 


\section{MODEL CONSTRUCTION}

\section{Selection of forecasting model}

(1)Annual electricity consumption forecasting

Gray forecast refers to an analysis, modeling, solving, forecasting process for the gray system. Grey Model Theory uses data generation means to weaken the system randomness(A.S. Khwaja et al. 2015). The original sequence shows a certain regularity properties. After the model construction it can also used to identify residual. Even if less historical data and random arbitrary distribution, higher prediction accuracy can also be obtained. Therefore, gray prediction has been widely used in agricultural planning, management decision-making, meteorology ecology, electricity demand forecasting, socio-economic and other sectors and industries(Chin Wang Lou and Ming Chui Dong. 2015).

As the original data of Guizhou is less and irregular, and in order to ensure the accuracy of long-term forecasting model, Grey prediction method has many advantages like requiring less original data, simple principle, not considering distribution, convenient operation, testable, being able to used for short-term forecasting, high prediction accuracy. Thus GM $(1,1)$ model is selected as the predictive model for Guizhou total electricity consumption(Yang Li et al. 2015).

(2)Monthly electricity consumption forecasting

Time series analysis and prediction method refers to speculate about the future trends by the use of past historical data and statistical analysis. In the process of analysis and forecasting, new performance and new features of data changes are analyzed, and these new performance and new features are taken full account in the predicted value. By studying seasonal time series, the relationship between the factors that affect the time series and time series can be better analyzed(Muhammad Qamar Raza and Abbas Khosravi. 2015). Meanwhile, the quarterly model has more observations than the annual model. The increase of the sample size makes that a lag structure with a longer lag period can be chosen in the modeling process. And it can explain the short-term economic cycle phenomenon which is difficult to be involved for year model, and it is very suitable for the analysis and prediction of short-period fluctuations of electricity. So policy makers can make more rational decisions in more authentic and full information set constraints(Hamed Chitsaz et al. 2015).

The annual duration load curve of electric power load shows obviously seasonal pattern. And it appears changes with natural season going. The peak value appears at a certain season each year, and the bottom value appears at other certain seasons(Irena Koprinska et al. 2015). But each peak and bottom values are not necessarily equal. In view of this, seasonal time series forecasting method can be used to predict the monthly electricity consumption in Guizhou.

\section{$\operatorname{GM}(1,1)$ Model}

GM $(1,1)$ model is a common gray forecasting model(Ming-Yue Zhai. 2015). It contains only univariate first order differential equations and it is an effective model for forecasting of power system(Zhongyi Hu et al. 2015). Its modeling steps are as follows:

Step 1: First order accumulated generating data sequence.

Giving original data series: $x^{(0)}(1), x^{(0)}(2), x^{(0)}(3), \ldots, x^{(0)}(n)$ meets $x^{(0)}(k) \geq 0 \quad k=1,2, \ldots, n$. A data sequence is generated

$$
x^{(1)}(k)=\sum_{i=1}^{k} x^{(0)}(i) \quad(k=1,2, \ldots, n)
$$

Step 2: Create differential equations

Sequences $x^{(1)}(k)$ has the law that is similar to exponential growth. And the solution form of first-order differential equations is also the exponential growth. Therefore, the sequence $x^{(1)}$ can be considered to meet the first-order linear differential equation model. 


$$
\frac{d x^{(1)}}{d t}+a x^{(1)}=u
$$

Where, $a$ is the coefficient for the model development reflecting the development trend of sequence and the original sequence. $u$ is coordination coefficient reflecting the transformation relationship between the data.

Step 3: Calculate the parameters $a$ and $u$

By the definition of the derivative, it can be obtained that:

$$
\frac{d x^{(1)}}{d t}=\lim _{\Delta t \rightarrow 0} \frac{x^{(1)}(t+\Delta t)-x^{(1)}(t)}{\Delta t}
$$

In terms of discrete form, differential term can be written as

$$
\frac{\Delta x^{(1)}}{\Delta t}=x^{(1)}(k+1)-x^{(1)}(k)=x^{(0)}(k+1)
$$

Where, $x^{(1)}$ can only be the mean of $k$ and $k+1$ at the same time, that is $0.5\left[x^{(1)}(k+1)+x^{(1)}(k)\right]$. Thus, the formula (2) can be rewritten as

$$
x^{(0)}(n)+0.5 a\left[x^{(1)}(n)+x^{(1)}(n-1)\right]=u
$$

Rewritten in matrix form, there is

$$
\left[\begin{array}{c}
x^{(0)}(2) \\
x^{(0)}(3) \\
\ldots \\
x^{(0)}(n)
\end{array}\right]=\left[\begin{array}{cc}
-0.5\left[x^{(1)}(1)+x^{(1)}(2)\right] & 1 \\
-0.5\left[x^{(1)}(2)+x^{(1)}(3)\right] & 1 \\
\ldots & \ldots \\
-0.5\left[x^{(1)}(n-1)+x^{(1)}(n)\right] & 1
\end{array}\right]\left(\begin{array}{l}
a \\
u
\end{array}\right)
$$

Abbreviated as

$$
\begin{gathered}
Y_{n}=B A \\
\text { Where, } \\
Y_{n}=\left[\begin{array}{c}
x^{(0)}(2) \\
x^{(0)}(3) \\
\cdots \\
x^{(0)}(n)
\end{array}\right], A=\left(\begin{array}{l}
a \\
u
\end{array}\right), \\
B=\left[\begin{array}{cc}
-0.5\left[x^{(1)}(1)+x^{(1)}(2)\right] & 1 \\
-0.5\left[x^{(1)}(2)+x^{(1)}(3)\right] & 1 \\
\cdots & \\
-0.5\left[x^{(1)}(n-1)+x^{(1)}(n)\right] & 1
\end{array}\right]
\end{gathered}
$$

The least squares estimation $\hat{a}$ of $a$ can be obtained:

$$
\hat{a}=\left[\begin{array}{l}
\hat{a} \\
\hat{u}
\end{array}\right]=\left(B^{T} B\right)^{-1} B^{T} Y_{n}
$$

Step 4: Create gray prediction model of original series $x^{(0)}$

Make $a$ and $u$ that are obtained back to the original differential equations, 


$$
\frac{d x^{(1)}}{d t}+\hat{a} x^{(1)}=\hat{u}
$$

It can be obtained that

$$
x^{(1)}(t+1)=\left[x^{(1)}(1)-\frac{\hat{u}}{\hat{a}}\right] e^{-\hat{a t}}+\frac{\hat{u}}{\hat{a}} \quad(k=0,1,2, \ldots)
$$

Make reduction for (10), and the Grey prediction model of the original series is

$$
\hat{x}^{(0)}(k+1)=\hat{x}^{(1)}(k+1)-\hat{x}^{(1)}(k)=\left(1-e^{\hat{a}}\right)\left(x^{(0)}(1)-\frac{\hat{u}}{\hat{a}}\right) e^{-\hat{a}^{*}} \quad(k=0,1,2, \ldots)
$$

\section{Seasonal time series model}

Seasonal time series show seasonal variation. These data have a similar change in the year. The calculation steps of seasonal coefficient method are as follows:

(1)Collect each quarter or each month time-series data of more than three years.

(2)Determine the arithmetic mean of all quarters or all months of the calendar

time series.

$$
\bar{X}=\frac{1}{n} \sum_{i=1}^{n} X_{i}
$$

year in the samples of

Where, is the number of time series sample data.

(3) Calculate arithmetic mean of time series data in the same month or same quarter.

(4) Determine quarter or month coefficient

$$
\beta_{i}=\frac{\bar{X}_{i}}{\bar{X}}
$$

Where, $i$ is the quarter or month number.

(5)Predict and calculation. When the time series are listed on a quarterly basis, firstly find the year weighted average prediction of prediction year (next year), that is

$$
y_{k+1}=\frac{W_{1} Y_{1}+W_{2} Y_{2}+\ldots+W_{k} Y_{k}}{W_{1}+W_{2}+\ldots+W_{k}}
$$

Where, $Y_{i}$ is the total value in the year $i ; W_{i}$ refers to the weight in the year $i$, and its value is according to the natural numbers; then calculate the quarterly average $\bar{y}_{k+1}$ of forecasting year, that is

$$
\bar{y}_{k+1}=\frac{y_{k+1}}{4}
$$

Then the forecasting value of the number $i$ quarter of the forecasting year is

$$
y_{k+1, i}=y_{k+1} \beta_{i}
$$

\section{EMPIRICAL ANALYSIS}

\section{Model Validation}

Combined with sold electric quantity data of each month in Guizhou from 2008 to 2012, forecast the sold electric quantity of each month in 2013. Compared with the actual sold electric quantity, the error analysis obtained is shown in table 1. 
Tab.1 Forecast sold electric quantity and error analysis in 2013

\begin{tabular}{|c|c|c|c|}
\hline Month & $\begin{array}{c}\text { Actual sole electric } \\
\text { quantity(ten } \\
\text { thousand } \mathrm{kWh})\end{array}$ & $\begin{array}{l}\text { Forecast sold } \\
\text { electric quantity } \\
(\text { ten thousand } \\
\text { kWh })\end{array}$ & $\begin{array}{l}\text { Relative } \\
\text { error(\%) }\end{array}$ \\
\hline 1 & 864396.01 & 858430.52 & $0.69 \%$ \\
\hline 2 & 738684.28 & 735149.20 & $0.48 \%$ \\
\hline 3 & 702459.94 & 707131.08 & $0.66 \%$ \\
\hline 4 & 746558.52 & 752485.40 & $0.79 \%$ \\
\hline 5 & 723644.72 & 719853.78 & $0.52 \%$ \\
\hline 6 & 744578.87 & 741718.54 & $0.38 \%$ \\
\hline 7 & 754662.05 & 758852.45 & $0.56 \%$ \\
\hline 8 & 796376.44 & 801000.44 & $0.58 \%$ \\
\hline 9 & 775869.46 & 769008.49 & $0.88 \%$ \\
\hline 10 & 760477.68 & 758698.20 & $0.23 \%$ \\
\hline 11 & 813664.52 & 818569.11 & $0.60 \%$ \\
\hline 12 & 815191.34 & 809287.15 & $0.72 \%$ \\
\hline
\end{tabular}

Seen from the table, combined with the data over the past five years, the relative error of forecast electric quantity and actual electric quantity in 2013 is less than $1 \%$ using gray prediction model and seasonal time series forecasting method. Therefore, this prediction method is applicable to forecast Guizhou sold electric quantity.

\section{Forecast Analysis}

Using gray prediction model and seasonal time series forecasting method, combined with the related sold electric quantity in Guizhou, forecast the sold electric quantity for each month from 2014 to 2016 , which is shown in table 2 .

Tab.2 Forecast sold electric quantity from 2014 to 2016

\begin{tabular}{cccc}
\hline & 2014 & 2015 & 2016 \\
\hline January & 863237.34 & 871254.42 & 879432.74 \\
February & 711760.26 & 723456.54 & 748743.12 \\
March & 756843.27 & 742143.65 & 753276.43 \\
April & 751416.81 & 758932.68 & 779532.06 \\
May & 736460.59 & 746587.57 & 757549.82 \\
June & 758625.09 & 762315.48 & 773467.40 \\
July & 776876.81 & 789043.58 & 790436.76 \\
August & 803211.83 & 810754.34 & 819263.54 \\
September & 791880.82 & 807436.24 & 814238.03 \\
October & 796415.92 & 791243.43 & 802541.67 \\
November & 820151.10 & 832145.45 & 840921.63 \\
December & 827778.60 & 834126.67 & 845625.54 \\
\hline
\end{tabular}

To get further comparative analysis of electric consumption characteristics of each month for three years from 2014 to 2016 in Guizhou, figure 1 is given. 


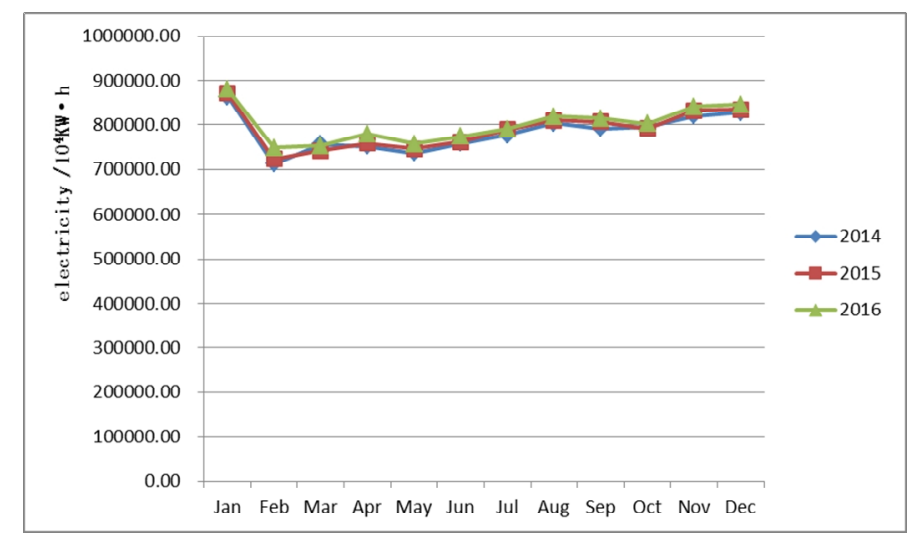

Fig. 1 Forecast sold electric quantity from 2014 to 2016

In order to analyze the grow trend of electric consumption of each month, the monthly growth rate of electricity consumption is shown in table 3.

Tab.3 Grow rate of forecast sold electric quantity from 2014 to 2016

\begin{tabular}{cccc}
\hline & 2014 & 2015 & 2016 \\
\hline January & $-0.13 \%$ & $0.93 \%$ & $0.94 \%$ \\
February & $-3.64 \%$ & $1.64 \%$ & $3.50 \%$ \\
March & $7.74 \%$ & $-1.94 \%$ & $1.50 \%$ \\
April & $0.65 \%$ & $1.00 \%$ & $2.71 \%$ \\
May & $1.77 \%$ & $1.38 \%$ & $1.47 \%$ \\
June & $1.89 \%$ & $0.49 \%$ & $1.46 \%$ \\
July & $2.94 \%$ & $1.57 \%$ & $0.18 \%$ \\
August & $0.86 \%$ & $0.94 \%$ & $1.05 \%$ \\
September & $2.06 \%$ & $1.96 \%$ & $0.84 \%$ \\
October & $4.73 \%$ & $-0.65 \%$ & $1.43 \%$ \\
November & $0.80 \%$ & $1.46 \%$ & $1.05 \%$ \\
December & $1.54 \%$ & $0.77 \%$ & $1.38 \%$
\end{tabular}

In order to reflect the trend of electricity of each month more directly, figure 2 is given.

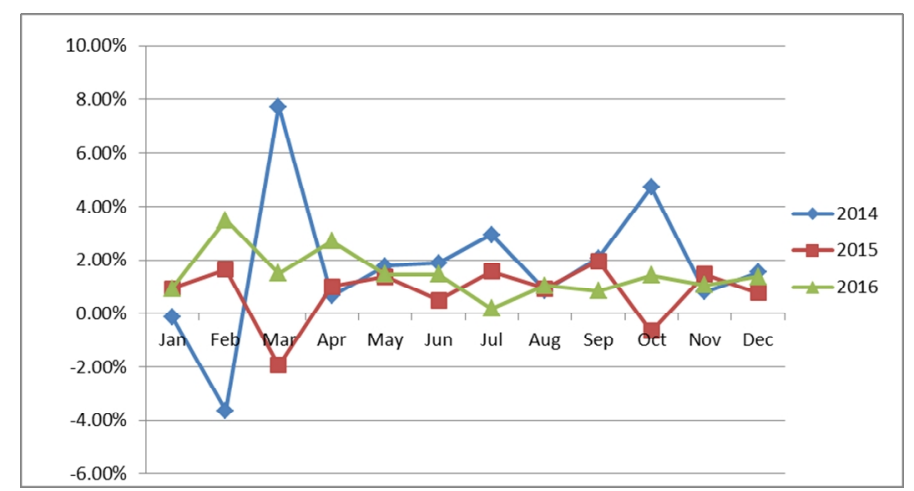

Fig. 2 Grow rate of forecast sold electric quantity from 2014 to 2016

\section{CONCLUSION}

This paper takes the advantages and disadvantages of gray prediction $\operatorname{GM}(1,1)$ and seasonal time series into account and proposes a combination electricity load forecasting model combined with GM(1,1) model and seasonal time series. Combined with related electric consumption data in 
Guizhou in recent years, make empirical analysis for the model proposed. The result shows that the combination electricity load forecasting model can predict electricity load of each month accurately. And it has reference value for achieving power grid security and economic operation.

\section{REFERENCES}

[1] A.S. Khwaja, M. Naeem, A. Anpalagan. 2015. Improved short-term load forecasting using bagged neural networks. Electric Power Systems Research 25: 109-115.

[2] Chin Wang Lou, Ming Chui Dong. 2015. A novel random fuzzy neural networks for tackling uncertainties of electric load forecasting. International Journal of Electrical Power \& Energy Systems 37: 33-44.

[3] Muhammad Qamar Raza, Abbas Khosravi. 2015. A review on artificial intelligence based load demand forecasting techniques for smart grid and buildings. Renewable and Sustainable Energy Reviews 50: 1352-1372.

[4] Hamed Chitsaz, Hamid Shaker, Hamidreza Zareipour. 2015. Short-term electricity load forecasting of buildings in microgrids. Energy and Buildings 15: 50-60.

[5] Yang Li, Yu-Qing Bao, Bin Yang. 2015. Modification method to deal with the accumulation effects for summer daily electric load forecasting. International Journal of Electrical Power \& Energy Systems 73: 913-918.

[6] Ming-Yue Zhai. 2015. A new method for short-term load forecasting based on fractal interpretation and wavelet analysis. International Journal of Electrical Power \& Energy Systems 69: 241-245.

[7] Irena Koprinska, Mashud Rana, Vassilios G. Agelidis. 2015. Correlation and instance based feature selection for electricity load forecasting. Knowledge-Based Systems 82: 29-40.

[8] Zhongyi Hu, Yukun Bao, Raymond Chiong. 2015. Mid-term interval load forecasting using multi-output support vector regression with a memetic algorithm for feature selection. Energy 84: 419-431. 